\title{
Textbook of Intensive and Acute Cardiac Care
}

\section{P. Ray}

Reçu le 14 février 2011 ; accepté le 4 mars 2011

(C) SFMU et Springer-Verlag France 2011

La Société européenne de cardiologie vient de publier un traité sur les pathologies cardiaques graves en réanimation (The ESCT textbook of Intensive and Acute Cardiac Care, Marco Tubaro, édition Oxford, New York, États-Unis, 2011, 810 pages). En s'entourant d'auteurs internationaux de renom (dont Nicolas Danchin et Gerasimos Filippatos comme coéditeurs et Patrick Goldstein, Pascal Vranckx et Doron Zahger comme éditeurs associés), Marco Tubaro réussit à aborder toutes les pathologies cardiaques aiguës graves, en particulier une large place est laissée à l'arrêt cardiaque, à l'insuffisance cardiaque, au syndrome coronarien et aussi aux troubles du rythme. Des rappels physiopathologiques, ainsi que la description des méthodes et des tests diagnostiques (dont imagerie et biomarqueurs) aident à une meilleure compréhension de la prise en charge actuelle des pathologies cardiaques aiguës graves. Ce traité devrait avoir un beau succès auprès des cardiologues et urgentistes.

Conflit d'intérêt : l'auteur ne déclare aucun conflit d'intérêt.
P. Ray $(\triangle)$

Service d'accueil des urgences,

groupe hospitalier Pitié-Salpêtrière,

47-83, boulevard de l'Hôpital,

université Pierre-et-Marie-Curie-Paris-VI,

F-75013 Paris, France

e-mail : patrick.ray@psl.aphp.fr 